\title{
COX inhibitors and sexual development
}

$\mathbf{T}$ he neural basis of sexual behavior is a subject of enduring interest and debate, which is likely to be further enlivened by the paper on page 643 of this issue. Amateau and McCarthy report that prostaglandin- $\mathrm{E}_{2}\left(\mathrm{PGE}_{2}\right)$ is required for the development of male-specific sexual behavior; male rats exposed during fetal or early neonatal life to cyclooxygenase (COX) inhibitors, which block $\mathrm{PGE}_{2}$ synthesis, show impaired copulatory behavior in adulthood, whereas female rats injected with $\mathrm{PGE}_{2}$ show male-like copulatory behavior. The involvement of $\mathrm{PGE}_{2}$ in male sexual differentiation is interesting and unexpected, as discussed in the accompanying News and Views (page 570). The question that will attract widest interest, however, is whether something similar could be happening in humans, given that COX inhibitors such as aspirin are often used by pregnant women.

The idea seems plausible on its face. COX inhibitors seem to affect sexual behavior by blocking downstream effects of testosterone, which is known to masculinize the developing brain through several different pathways. One of its targets is the preoptic area, which is closely associated with the hypothalamus and which contains sexually dimorphic structures in both rodents and humans. Lesions to the POA disrupt sexual behavior, and Amateau and McCarthy have shown that the behavioral effects of their drug treatments are accompanied by morphological changes in the POA.

It is of course difficult to extrapolate from rat copulation to the much greater complexity of human sexuality. Libido, sexual orientation and gender identity, for example, seem to be distinct and dissociable traits in humans, and although gonadal steroids probably influence all these behaviors, they are likely to do so through distinct neural structures, and possibly through distinct biochemical pathways. The behavioral effect in Amateau and McCarthy's study seems to reflect an alteration of libido rather than sexual preference (which was not tested directly), so there is no reason at this point to suspect that COX inhibitors contribute to homosexuality in humans.

Nevertheless, if prenatal exposure to COX inhibitors does affect some aspect of human sexuality, this could easily have escaped notice. The lag from cause to effect could be several decades, and there was no previous reason to look for any such link. Now that there is a motivation for doing so, the only way to test the hypothesis is through a longitudinal epidemiological study. These tests are by necessity longterm projects, but at least one such study is already underway. The Avon Longitudinal Study of Parents and Children (ALSPAC) was initiated about 15 years ago by Jean Golding, an epidemiologist at Bristol University, with the aim of studying genetic and early environmental effects on health and other life outcomes. Golding and her colleagues recruited a cohort of almost 15,000 pregnant women, whose children, born in 1991-1992, have been tracked ever since. The researchers took blood samples from the pregnant mothers and also gathered information about drug use, including commonly used COX inhibitors such as aspirin and Paracetamol (acetaminophen, sold as Tylenol in the USA). About half the mothers reported taking Paracetemol during pregnancy, and the ALSPAC group has found that high usage is associated with wheezing in the children ${ }^{1}$, suggesting a possible link to asthma.

The same cohort can also be used to test for sexual effects. Among the researchers collaborating with Golding is Melissa Hines at City University in London, an expert on the neural basis of human sexual behavior. Hines has already reported, based on ALSPAC data, that gender-role behavior in three and a half-year-olds is associated with maternal testosterone levels; girls who are 'tomboys' (for example, preferring toy trucks to dolls) are more likely to have been exposed to high levels of testosterone in utero than those whose behavior is more sex-typical ${ }^{2}$. Hines is reexamining these data to see whether there is any relationship between gender role behavior and exposure to COX inhibitors. She also plans to conduct a follow-up study with the children, who are now approaching 13 . Adolescents can be reticent about discussing sexual matters, but the questionnaires are computerized and strictly anonymous, so Hines is optimistic that it will be possible to obtain meaningful data about sexual relationships and orientation, as well as longer-term outcomes such as marriage and fertility.

Until these epidemiological studies are completed, the possible effect of COX inhibitors on humans must remain a matter for speculation. In the meantime, while caution seems appropriate, there is no cause for panic. Examples such as thalidomide and DES (a synthetic estrogen that has been associated with cancer and infertility in exposed female children) are well known, and the possibility that another common class of drugs could affect fetal development is bound to cause concern for many expectant parents, but one must always be careful when extrapolating from rodents to humans. Indeed DES is a case in point: in many species it causes strong masculinization of female behavior, but its effect on humans is very subtle. In particular, earlier claims of an association between DES exposure and lesbianism have not been replicated.

COX inhibitors are used to treat several serious conditions during pregnancy, and for some clinical situations they may still be the best option. Nevertheless, the prudent advice is that it is always best to avoid unnecessary medication during pregnancy; the new findings reinforce this point by providing a sharp reminder of one of the most fundamental principles in science, namely the law of unintended consequences.

\footnotetext{
1. Shaheen S.O. et al. Thorax 57, 958-963 (2002).

2. Hines, M. et al. Child Dev. 73, 1678-1687 (2002).
} 